\title{
A SECOND ORDER THREE-POINT BOUNDARY VALUE PROBLEM WITH MIXED NONLINEAR BOUNDARY CONDITIONS*
}

\author{
BASHIR AHMAD ${ }^{\dagger}$ AND TAGREED G. SOGATI ${ }^{\ddagger}$
}

\begin{abstract}
We apply the generalized quasilinearization method to a second order three-point boundary value problem involving mixed nonlinear boundary conditions and obtain a monotone sequence of approximate solutions converging to the unique solution of the problem possessing a convergence of order $k(k \geq 2)$.
\end{abstract}

Key words. Quasilinearization, Three-point boundary value problem, Rapid convergence

AMS subject classifications. 34A37, 34B15

1. Introduction. The method of quasilinearization developed by Bellman and Kalaba [1] and generalized by Lakshmikantham [2-3] later on, has been studied and extended in several diverse disciplines. In fact, it is generating a rich history and an extensive bibliography can be found in [4-10].

Multi-point nonlinear boundary value problems, which refer to a different family of boundary conditions in the study of disconjugacy theory [11], have been addressed by many authors, for example, see [12-14]. In particular, Eloe and Gao [15] discussed the quasilinearization method for a three-point boundary value problem. In this paper, we study the generalized quasilinearization method for a second order three-point boundary value problem with mixed nonlinear boundary conditions. In fact, a sequence of approximate solutions converging monotonically to a solution of the nonlinear three-point problem with the order of convergence $k(k \geq 2)$ has been presented.

2. Preliminary results. Consider a three-point boundary value problem with mixed nonlinear boundary conditions

$$
\begin{gathered}
x^{\prime \prime}(t)=f(t, x(t)), \\
p x(0)-q x^{\prime}(0)=a, \quad p x(1)+q x^{\prime}(1)=g\left(x\left(\frac{1}{2}\right)\right),
\end{gathered}
$$

where $f$ is continuous with $f_{x}>0$ on $[0,1] \times R, \quad p, q>0$ with $p>1$ and $g: R \longrightarrow R$ is continuous. By Green's function method, the solution, $x(t)$ of (1.1)-(1.2) can be written as

$$
x(t)=a\left(\frac{-t}{p+2 q}+\frac{p+q}{p^{2}+2 p q}\right)+g\left(x\left(\frac{1}{2}\right)\right)\left[\frac{t}{p+2 q}+\frac{q}{p^{2}+2 p q}\right]+\int_{0}^{1} G(t, s) f(s, x(s)) d s,
$$

where the Green's function $G(t, s)$ for the mixed three-point boundary value problem is given by

$$
G(t, s)=\frac{1}{\left(p^{2}+2 p q\right)} \begin{cases}(p t+q)(p(s-1)-q), & \text { if } 0 \leq t \leq s \leq 1 \\ (p(t-1)-q)(p s+q), & \text { if } 0 \leq s \leq t \leq 1\end{cases}
$$

\footnotetext{
*Received April 6, 2004; accepted for publication December 3, 2004.

${ }^{\dagger}$ Department of Mathematics, Faculty of Science, King Abdul Aziz University, P.O. Box 80203, Jeddah 21589, Saudi Arabia (bashir_qau@yahoo.com).

${ }_{\ddagger}^{\ddagger}$ Department of Mathematics, Faculty of Science, King Abdul Aziz University, P.O. Box 80203, Jeddah 21589, Saudi Arabia (teetawa@hotmail.com).
} 
Notice that $G(t, s)<0$ on $[0,1] \times[0,1]$. (1.2) if

We say that $\alpha \in C^{2}[0,1]$ is a lower solution of the boundary value problem (1.1)-

$$
\begin{gathered}
\alpha^{\prime \prime}(t) \geq f(t, \alpha), t \in[0,1], \\
p \alpha(0)-q \alpha^{\prime}(0) \leq a, \quad p \alpha(1)+q \alpha^{\prime}(1) \leq g\left(\alpha\left(\frac{1}{2}\right)\right),
\end{gathered}
$$

and $\beta \in C^{2}[0,1]$ is an upper solution of the boundary value problem (1.1)-(1.2) if

$$
\begin{gathered}
\beta^{\prime \prime}(t) \leq f(t, \beta), t \in[0,1], \\
p \beta(0)-q \beta^{\prime}(0) \geq a, \quad p \beta(1)+q \beta^{\prime}(1) \geq g\left(\beta\left(\frac{1}{2}\right)\right) .
\end{gathered}
$$

Theorem 1. Assume that $f$ is continuous with $f_{x}>0$ on $[0,1] \times R$ and $g$ is continuous with $0 \leq g^{\prime}<1$ on $R$. Let $\beta$ and $\alpha$ be the upper and lower solutions of (1.1)-(1.2) respectively. Then $\alpha(t) \leq \beta(t), t \in[0,1]$.

Proof. Define $h(t)=\alpha(t)-\beta(t)$. For the sake of contradiction, we suppose that $h(t)>0$ for some $t \in[0,1]$. First we take $t_{0} \in(0,1)$. Then by the definition of lower and upper solutions together with $f_{x}>0$, we obtain

$$
h^{\prime \prime}\left(t_{0}\right)=\alpha^{\prime \prime}\left(t_{0}\right)-\beta^{\prime \prime}\left(t_{0}\right) \geq f\left(t_{0}, \alpha\left(t_{0}\right)\right)-f\left(t_{0}, \beta\left(t_{0}\right)\right)>0 .
$$

By the standard methodology, let $h(t)$ have a local positive maximum at $t_{0} \in(0,1)$, then $h^{\prime}\left(t_{0}\right)=0$ and $h^{\prime \prime}\left(t_{0}\right) \leq 0$, which contradicts (1.3). Thus, for $t_{0} \in(0,1)$, we have $\alpha(t) \leq \beta(t)$. Now, suppose that $h(t)$ has a local positive maximum at $t_{0}=1$, then $h^{\prime}(1)=0$ and $h^{\prime \prime}(1)<0$. On the other hand, by definition of lower and upper solutions and in view of the condition $0 \leq g^{\prime}<1$, we find that

$$
\begin{aligned}
p h(1)+q h^{\prime}(1) & \leq g\left(\alpha\left(\frac{1}{2}\right)\right)-g\left(\beta\left(\frac{1}{2}\right)\right) \\
& =\frac{g\left(\alpha\left(\frac{1}{2}\right)\right)-g\left(\beta\left(\frac{1}{2}\right)\right)}{\alpha\left(\frac{1}{2}\right)-\beta\left(\frac{1}{2}\right)}\left[\alpha\left(\frac{1}{2}\right)-\beta\left(\frac{1}{2}\right)\right] \\
& \leq \alpha\left(\frac{1}{2}\right)-\beta\left(\frac{1}{2}\right) \\
& =h\left(\frac{1}{2}\right) .
\end{aligned}
$$

Thus, $p h(1) \leq h\left(\frac{1}{2}\right)$ or $h(1)<h\left(\frac{1}{2}\right)$ for $p>1$, which is a contradiction. Similarly, we get a contradiction for $t_{0}=0$. Hence we conclude that $\alpha(t) \leq \beta(t)$ on $[0,1]$.

Theorem 2. Assume that $f$ is continuous on $[0,1] \times R$ with $f_{x}>0$ and $g$ is continuous on $R$ satisfying $0 \leq g^{\prime}<1$. Further, we assume that there exist an upper solution $\beta$ and a lower solution $\alpha$ of (1.1)-(1.2) such that $\alpha(t) \leq \beta(t), t \in[0,1]$. Then 
there exists a solution $x(t)$ of (1.1)-(1.2) satisfying $\alpha(t) \leq x(t) \leq \beta(t), t \in[0,1]$.

Proof. Define $F$ and $G$ by

$$
\begin{gathered}
F(t, x)=\left\{\begin{array}{cc}
f(t, \beta)+\frac{x-\beta}{1+x-\beta}, & \text { if } x(t)>\beta(t), \\
f(t, x), & \text { if } \alpha(t) \leq x(t) \leq \beta(t), \\
f(t, \alpha)+\frac{x-\alpha}{1+|x-\alpha|}, & \text { if } x(t)<\alpha(t),
\end{array}\right. \\
G(x)=\left\{\begin{array}{cc}
g\left(\beta\left(\frac{1}{2}\right)\right), & \text { if } x>\beta\left(\frac{1}{2}\right), \\
g(x), & \text { if } \alpha\left(\frac{1}{2}\right) \leq x \leq \beta\left(\frac{1}{2}\right), \\
g\left(\alpha\left(\frac{1}{2}\right)\right), & \text { if } x<\alpha\left(\frac{1}{2}\right) .
\end{array}\right.
\end{gathered}
$$

Since $F(t, x)$ and $G(x)$ are continuous and bounded, a standard application of Schauder's fixed point theorem ensures the existence of a solution, $x$ of the problem

$$
\begin{gathered}
x^{\prime \prime}(t)=F(t, x(t)), t \in[0,1], \\
p x(0)-q x^{\prime}(0)=a, \quad p x(1)+q x^{\prime}(1)=G\left(x\left(\frac{1}{2}\right)\right) .
\end{gathered}
$$

In order to complete the proof, we need to show that $\alpha(t) \leq x(t) \leq \beta(t)$ on $[0,1]$ which can be done using the procedure employed in the proof of theorem 1 . In this case, $G$ satisfies $0 \leq G^{\prime} \leq 1$ on $\left[\alpha\left(\frac{1}{2}\right), \beta\left(\frac{1}{2}\right)\right]$.

REMARK. In case of the problem $-x^{\prime \prime}(t)=f(t, x(t))$, we require the condition $f_{x}<0$ and the corresponding Green's function $G(t, s)$ is nonnegative, that is,

$$
G(t, s) \geq \frac{q^{2}}{\left(p^{2}+2 p q\right)},(t, s) \in[0,1] \times[0,1] .
$$

\section{Main result.}

Theorem 3. Assume that

$\left(\mathbf{A}_{1}\right) \frac{\partial^{i}}{\partial x^{i}} f(t, x), i=0,1,2, \ldots, k$, are continuous on $[0,1] \times R$ satisfying $\frac{\partial^{i}}{\partial x^{i}} f(t, x) \geq$ $0, i=0,1,2, \ldots, k-1$, with $\frac{\partial^{k}}{\partial x^{k}}(f(t, x)+\phi(t, x)) \leq 0$, where $\frac{\partial^{i}}{\partial x^{i}} \phi(t, x), i=$ $0,1,2, \ldots, k$ are continuous and $\frac{\partial^{k}}{\partial x^{k}} \phi(t, x) \leq 0$ for some function $\phi(t, x)$.

$\left.\left(\mathbf{A}_{\mathbf{2}}\right) \alpha, \beta \in C^{2}[0,1], R\right]$ are lower and upper solutions of (1.1)-(1.2) respectively.

$\left(\mathbf{A}_{3}\right) \frac{d^{i}}{d x^{i}} g(x), \quad i=0,1,2, \ldots, k$, are continuous on $R$ satisfying $0 \leq \frac{d^{i}}{d x^{i}} g(x)<$ $\frac{M}{(\beta-\alpha)^{i-1}}$ with $\frac{d^{k}}{d x^{k}} g(x) \geq 0$ and $0<M<\frac{1}{3}$.

Then there exists a monotone sequence of approximate solutions $\left\{w_{n}\right\}$ converging to the unique solution, $x$ of (1.1)-(1.2) with the order of convergence $k(k \geq 2)$.

Proof. Define $F:[0,1] \times R \longrightarrow R$ by

$$
F(t, x)=f(t, x)+\phi(t, x) .
$$

Using $\left(A_{1}\right),\left(A_{3}\right)$ and the generalized mean value theorem, we obtain

$$
f(t, x) \leq \sum_{i=0}^{k-1} \frac{\partial^{i}}{\partial x^{i}} F(t, y) \frac{(x-y)^{i}}{i !}-\phi(t, x),
$$




$$
g(x) \geq \sum_{i=0}^{k-1} \frac{d^{i}}{d x^{i}} g(y) \frac{(x-y)^{i}}{i !} .
$$

Set

$$
F^{* *}(t, x, y)=\sum_{i=0}^{k-1} \frac{\partial^{i}}{\partial x^{i}} F(t, y) \frac{(x-y)^{i}}{i !}-\phi(t, x)
$$

and

$$
h^{*}(x, y)=\sum_{i=0}^{k-1} \frac{d^{i}}{d x^{i}} g(y) \frac{(x-y)^{i}}{i !} .
$$

Observe that $F^{* *}(t, x, y)$ and $h^{*}(x, y)$ are continuous and

$$
\begin{array}{cl}
f(t, x)=\min _{y} F^{* *}(t, x, y), & f(t, x)=F^{* *}(t, x, x), \\
g(x)=\max _{y} h^{*}(x, y), & g(x)=h^{*}(x, x) .
\end{array}
$$

Expanding $\phi(t, x)$ by Taylor's theorem, (1.4) takes the form

$$
F^{* *}(t, x, y)=\sum_{i=0}^{k-1} \frac{\partial^{i}}{\partial x^{i}} f(t, y) \frac{(x-y)^{i}}{i !}-\frac{\partial^{k}}{\partial x^{k}} \phi(t, \xi) \frac{(x-y)^{k}}{k !} .
$$

Differentiating (1.8) and using $\left(A_{1}\right)$, we get

$$
F_{x}^{* *}(t, x, y)>\sum_{i=1}^{k-1} \frac{\partial^{i}}{\partial x^{i}} f(t, y) \frac{(x-y)^{i-1}}{(i-1) !} \geq 0,
$$

which implies that $F_{x}^{* *}(t, x, y)$ is increasing in $x$ for each $(t, y) \in[0,1] \times R$. Similarly, differentiation of (1.5) together with $\left(A_{3}\right)$ yields

$$
h_{x}^{*}(x, y)=\sum_{i=1}^{k-1} \frac{d^{i}}{d x^{i}} g(y) \frac{(x-y)^{i-1}}{(i-1) !},
$$

which is clearly nonnegative and further

$$
\begin{aligned}
h_{x}^{*}(x, y) & =\sum_{i=1}^{k-1} \frac{d^{i}}{d x^{i}} g(y) \frac{(x-y)^{i-1}}{(i-1) !} \\
& \leq \sum_{i=1}^{k-1} \frac{d^{i}}{d x^{i}} g(y) \frac{(\beta-\alpha)^{i-1}}{(i-1) !} \\
& \leq \sum_{i=1}^{k-1} \frac{M}{(i-1) !}<M\left(1+\sum_{i=1}^{k-2} \frac{1}{2^{i-1}}\right)=M\left(3-\frac{1}{2^{k-3}}\right) \\
& <3 M<1
\end{aligned}
$$

where $\alpha \leq y \leq x \leq \beta$. Select $\alpha=w_{0}$ and consider the following mixed problem

$$
x^{\prime \prime}=F^{* *}\left(t, x(t), w_{0}(t)\right), t \in[0,1],
$$




$$
p x(0)-q x^{\prime}(0)=a, \quad p x(1)+q x^{\prime}(1)=h^{*}\left(x\left(\frac{1}{2}\right), w_{0}\left(\frac{1}{2}\right)\right) .
$$

Using $\left(A_{3}\right),(1.6)$ and (1.7), we obtain

$$
\begin{gathered}
w_{0}^{\prime \prime} \geq f\left(t, w_{0}\right)=F^{* *}\left(t, w_{0}, w_{0}\right), t \in[0,1], \\
p w_{0}(0)-q w_{0}^{\prime}(0) \leq a, \quad p w_{0}(1)+q w_{0}^{\prime}(1) \leq g\left(w_{0}\left(\frac{1}{2}\right)\right)=h^{*}\left(w_{0}\left(\frac{1}{2}\right), w_{0}\left(\frac{1}{2}\right)\right),
\end{gathered}
$$

and

$$
\begin{gathered}
\beta^{\prime \prime} \leq f(t, \beta) \leq F^{* *}\left(t, \beta, w_{0}\right), t \in[0,1], \\
p \beta(0)-q \beta^{\prime}(0) \geq a, \quad p \beta(1)+q \beta^{\prime}(1) \geq g\left(\beta\left(\frac{1}{2}\right)\right) \geq h^{*}\left(\beta\left(\frac{1}{2}\right), w_{0}\left(\frac{1}{2}\right)\right),
\end{gathered}
$$

which imply that $w_{0}$ and $\beta$ are lower and upper solutions of (1.10)-(1.11) respectively. It follows by Theorems 1 and 2 that there exists a unique solution, $w_{1}$ of (1.10)-(1.11) such that

$$
w_{0}(t) \leq w_{1}(t) \leq \beta(t), t \in[0,1] .
$$

Now, we consider the problem

$$
\begin{gathered}
x^{\prime \prime}=F^{* *}\left(t, x(t), w_{1}(t)\right), t \in[0,1], \\
p x(0)-q x^{\prime}(0)=a, \quad p x(1)+q x^{\prime}(1)=h^{*}\left(x\left(\frac{1}{2}\right), w_{1}\left(\frac{1}{2}\right)\right) .
\end{gathered}
$$

Again, using $\left(A_{3}\right),(1.6)$ and $(1.7)$, we get

$$
w_{1}^{\prime \prime}=F^{* *}\left(t, w_{1}, w_{0}\right) \geq F^{* *}\left(t, w_{1}, w_{1}\right), t \in[0,1]
$$

$p w_{1}(0)-q w_{1}^{\prime}(0) \leq a, \quad p w_{1}(1)+q w_{1}^{\prime}(1)=h^{*}\left(w_{1}\left(\frac{1}{2}\right), w_{0}\left(\frac{1}{2}\right)\right) \leq h^{*}\left(w_{1}\left(\frac{1}{2}\right), w_{1}\left(\frac{1}{2}\right)\right)$,

and

$$
\begin{gathered}
\beta^{\prime \prime} \leq f(t, \beta) \leq F^{* *}\left(t, \beta, w_{1}\right), t \in[0,1], \\
p \beta(0)-q \beta^{\prime}(0) \geq a, \quad p \beta(1)+q \beta^{\prime}(1) \geq g\left(\beta\left(\frac{1}{2}\right)\right) \geq h^{*}\left(\beta\left(\frac{1}{2}\right), w_{1}\left(\frac{1}{2}\right)\right),
\end{gathered}
$$

implying that $w_{1}$ and $\beta$ are lower and upper solutions of (1.12) - (1.13) respectively. By the earlier arguments, we find a solution, $w_{2}$ of (1.12) - (1.13) such that

$$
w_{0}(t) \leq w_{2}(t) \leq \beta(t), t \in[0,1] .
$$

Continuing this process successively, we obtain a monotone sequence $\left\{w_{n}\right\}$ of solutions satisfying

$$
w_{0}(t) \leq w_{1}(t) \leq w_{2}(t) \leq \ldots \leq w_{n}(t) \leq \beta(t), t \in[0,1]
$$


where each element $w_{n}$ of the sequence is a solution of the following problem

$$
\begin{gathered}
x^{\prime \prime}=F^{* *}\left(t, x(t), w_{n-1}(t)\right), t \in[0,1], \\
p x(0)-q x^{\prime}(0)=a, \quad p x(1)+q x^{\prime}(1)=h^{*}\left(x\left(\frac{1}{2}\right), w_{n-1}\left(\frac{1}{2}\right)\right),
\end{gathered}
$$

and is given by

$$
\begin{aligned}
w_{n}(t) & =a\left(\frac{-t}{p+2 q}+\frac{p+q}{p^{2}+2 p q}\right)+h^{*}\left(w_{n}\left(\frac{1}{2}\right), w_{n-1}\left(\frac{1}{2}\right)\right)\left[\frac{t}{p+2 q}+\frac{q}{p^{2}+2 p q}\right] \\
& +\int_{0}^{1} G(t, s) F^{* *}\left(s, w_{n}, w_{n-1}\right) d s .
\end{aligned}
$$

In view of the fact that $[0,1]$ is compact and the monotone convergence is pointwise, it follows that the convergence of the sequence is uniform. If $x(t)$ is the limit point of the sequence, then passing onto the limit $n \rightarrow \infty$, (1.14) gives

$$
\begin{aligned}
x(t) & =a\left(\frac{-t}{p+2 q}+\frac{p+q}{p^{2}+2 p q}\right)+h^{*}\left(x\left(\frac{1}{2}\right), x\left(\frac{1}{2}\right)\right)\left[\frac{t}{p+2 q}+\frac{q}{p^{2}+2 p q}\right] \\
& +\int_{0}^{1} G(t, s) F^{* *}(s, x(s), x(s)) d s \\
& =a\left(\frac{-t}{p+2 q}+\frac{p+q}{p^{2}+2 p q}\right)+g\left(x\left(\frac{1}{2}\right)\right)\left[\frac{t}{p+2 q}+\frac{q}{p^{2}+2 p q}\right] \\
& +\int_{0}^{1} G(t, s) f(s, x(s)) d s .
\end{aligned}
$$

Thus $x(t)$ is the solution of (1.1)-(1.2).

Now, we show that the convergence of the sequence of iterates is of order $k(k \geq 2)$. For that, we set $e_{n}(t)=x(t)-w_{n}(t), a_{n}(t)=w_{n+1}(t)-w_{n}(t), t \in[0,1]$ and note that $e_{n}(t) \geq 0, a_{n}(t) \geq 0, e_{n}(t)-a_{n}(t)=e_{n+1}(t)$. Also $e_{n}(t) \geq a_{n}(t)$ and hence by induction $e_{n}^{k}(t) \geq a_{n}^{k}(t)$. Further

$$
p e_{n}(0)-q e_{n}^{\prime}(0)=0, p e_{n}(1)+q e_{n}^{\prime}(1)=g\left(x\left(\frac{1}{2}\right)\right)-h^{*}\left(w_{n}\left(\frac{1}{2}\right), w_{n-1}\left(\frac{1}{2}\right)\right) .
$$

Using the generalized mean value theorem, we have

$$
\begin{aligned}
e_{n+1}^{\prime \prime}(t) & =x^{\prime \prime}-w_{n+1}^{\prime \prime} \\
& =\sum_{i=0}^{k-1} \frac{\partial^{i}}{\partial x^{i}} f\left(t, w_{n}\right) \frac{\left(x-w_{n}\right)^{i}}{i !}+\frac{\partial^{k}}{\partial x^{k}} f(t, \xi) \frac{\left(x-w_{n}\right)^{k}}{k !} \\
& -\sum_{i=0}^{k-1} \frac{\partial^{i}}{\partial x^{i}} f\left(t, w_{n}\right) \frac{\left(w_{n+1}-w_{n}\right)^{i}}{i !}+\frac{\partial^{k}}{\partial x^{k}} \phi(t, \xi) \frac{\left(w_{n+1}-w_{n}\right)^{k}}{k !} \\
& =\sum_{i=1}^{k-1} \frac{\partial^{i}}{\partial x^{i}} f\left(t, w_{n}\right) \frac{\left(e_{n}^{i}-a_{n}^{i}\right)}{i !}+\frac{\partial^{k}}{\partial x^{k}} f(t, \xi) \frac{\left(e_{n}\right)^{k}}{k !}+\frac{\partial^{k}}{\partial x^{k}} \phi(t, \xi) \frac{\left(a_{n}\right)^{k}}{k !} \\
& \geq\left(\sum_{i=1}^{k-1} \frac{\partial^{i}}{\partial x^{i}} f\left(t, w_{n}\right) \frac{1}{i !} \sum_{j=0}^{k-1} e_{n}^{j} a_{n}^{i-1-j}\right) e_{n+1}+\left(\frac{\partial^{k}}{\partial x^{k}} f(t, \xi)+\frac{\partial^{k}}{\partial x^{k}} \phi(t, \xi)\right) \frac{\left(e_{n}\right)^{k}}{k !} \\
& \geq \frac{\partial^{k}}{\partial x^{k}} F(t, \xi) \frac{\left(e_{n}\right)^{k}}{k !} \geq-M \|\left. e_{n}\right|^{k},
\end{aligned}
$$


where $\mathrm{M}$ is a bound on $\frac{1}{k !} \frac{\partial^{k}}{\partial x^{k}} F(t, \xi)$ for $t \in[0,1]$. Thus, in view of (1.15), we have

$$
\begin{aligned}
e_{n+1}(t) & =\left(g\left(x\left(\frac{1}{2}\right)\right)-h^{*}\left(w_{n+1}\left(\frac{1}{2}\right), w_{n}\left(\frac{1}{2}\right)\right)\right)\left[\frac{t}{p+2 q}+\frac{q}{p^{2}+2 p q}\right]+\int_{0}^{1} G(t, s) e_{n+1}^{\prime \prime}(t) d s \\
\leq & \left(g\left(x\left(\frac{1}{2}\right)\right)-h^{*}\left(w_{n+1}\left(\frac{1}{2}\right), w_{n}\left(\frac{1}{2}\right)\right)\right)\left[\frac{t}{p+2 q}+\frac{q}{p^{2}+2 p q}\right] \\
& +M\left\|e_{n}\right\|^{k} \int_{0}^{1}|G(t, s)| d s \\
& =\left[\sum_{i=0}^{k-1} \frac{d^{i}}{d x^{i}} g\left(w_{n}\left(\frac{1}{2}\right)\right) \frac{\left(x\left(\frac{1}{2}\right)-w_{n}\left(\frac{1}{2}\right)\right)^{i}}{i !}+\frac{d^{k}}{d x^{k}} g\left(\xi\left(\frac{1}{2}\right)\right) \frac{\left(x\left(\frac{1}{2}\right)-w_{n}\left(\frac{1}{2}\right)\right)^{k}}{k !}\right. \\
& \left.-\sum_{i=0}^{k-1} \frac{d^{i}}{d x^{i}} g\left(w_{n}\left(\frac{1}{2}\right)\right) \frac{\left(w_{n+1}\left(\frac{1}{2}\right)-w_{n}\left(\frac{1}{2}\right)\right)^{i}}{i !}\right]\left[\frac{t}{p+2 q}+\frac{q}{p^{2}+2 p q}\right] \\
& +M_{1}\left\|e_{n}\right\|^{k} \\
& =\left[\sum_{i=1}^{k-1} \frac{d^{i}}{d x^{i}} g\left(w_{n}\left(\frac{1}{2}\right)\right) \frac{\left(e_{n}^{i}\left(\frac{1}{2}\right)-a_{n}^{i}\left(\frac{1}{2}\right)\right)}{i !}+\frac{d^{k}}{d x^{k}} g\left(\xi\left(\frac{1}{2}\right)\right) \frac{\left(e_{n}\left(\frac{1}{2}\right)\right)^{k}}{k !}\right]\left[\frac{t}{p+2 q}\right. \\
+ & \left.\frac{q}{p^{2}+2 p q}\right]+M_{1}\left\|e_{n}\right\|^{k} \\
= & {\left[\sum_{i=1}^{k-1} \frac{d^{i}}{d x^{i}} g\left(w_{n}\left(\frac{1}{2}\right)\right) \frac{1}{i !} \sum_{j=0}^{k-1} e_{n}^{j}\left(\frac{1}{2}\right) a_{n}^{i-1-j}\left(\frac{1}{2}\right) e_{n+1}\left(\frac{1}{2}\right)\right.} \\
+ & \left.\frac{d^{k}}{d x^{k}} g\left(\xi\left(\frac{1}{2}\right)\right) \frac{\left(e_{n}\left(\frac{1}{2}\right)\right)^{k}}{k !}\right]\left[\frac{t}{p+2 q}+\frac{q}{p^{2}+2 p q}\right]+M_{1}\left\|e_{n}\right\|^{k} \\
\leq & {\left[\sum_{i=0}^{k-1} \frac{M}{(\beta-\alpha)^{i-1}} \frac{1}{i !} \sum_{j=0}^{i-1} e_{n}^{i-1-j}\left(\frac{1}{2}\right) a_{n}^{j}\left(\frac{1}{2}\right)\right] M_{3} e_{n+1}\left(\frac{1}{2}\right)+M_{2} M_{3}\left\|e_{n}\right\|^{k}+M_{1}\left\|e_{n}\right\|^{k} . }
\end{aligned}
$$

where $M_{1}$ provides a bound for $M \int_{0}^{1}|G(t, s)| d s, \quad M_{2}$ provides a bound for $\frac{d^{k}}{d x^{k}} g\left(\xi\left(\frac{1}{2}\right)\right) \frac{1}{k !}$, and $M_{3}=\frac{1}{p+2 q}+\frac{q}{p^{2}+2 p q}$. Letting

$$
P_{n}(t)=\sum_{i=0}^{k-1} \frac{M}{(\beta-\alpha)^{i-1}} \frac{1}{i !} \sum_{j=0}^{i-1} e_{n}^{i-1-j}\left(\frac{1}{2}\right) a_{n}^{j}\left(\frac{1}{2}\right),
$$

we find that

$$
\lim _{n \rightarrow \infty} P_{n}(t)=\lim _{n \rightarrow \infty} \sum_{i=0}^{k-1} \frac{M}{(\beta-\alpha)^{i-1}} \frac{1}{i !} \sum_{j=0}^{i-1} e_{n}^{i-1-j}\left(\frac{1}{2}\right) a_{n}^{j}\left(\frac{1}{2}\right)=M<\frac{1}{3} .
$$

Therefore, we can choose $\lambda<\frac{1}{3}$ and $n_{0} \in N$ such that for $n \geq n_{0}$, we have $P_{n}(t)<\lambda$ and consequently (1.16) becomes

$$
\left\|e_{n+1}\right\|<\lambda_{1}\left\|e_{n+1}\right\|+M_{4}\left\|e_{n}\right\|^{k} .
$$

Solving (1.17) algebraically yields

$$
\left\|e_{n+1}\right\| \leq \frac{M_{4}}{1-\lambda_{1}}\left\|e_{n}\right\|^{k}
$$


where $M_{4}=M_{1}+M_{2} M_{3}, \quad \lambda_{1}=\lambda M_{3}$ and $\left\|e_{n}\right\|=\max \left\{\left|e_{n}(t)\right|: t \in[0,1]\right\}$ is the usual uniform norm. This completes the proof.

EXAMPLE. As an example, we can take $f(t, x)=e^{x}$ and $g(x)=x^{p}$ (for instance, $p=k)$ in (1.1)-(1.2) which clearly satisfy the hypotheses of the main result.

Acknowledgement. The authors gratefully acknowledge the referee for his/her useful suggestions to improve the quality of the paper.

\section{REFERENCES}

[1] R.Bellamn and R. Kalaba, Quasilinearization and Nonlinear Boundary Value Problems, Amer. Elsevier, New York, 1965.

[2] V. Lakshmikantham, An extension of the method of quasilinearization, J. Optim. Theory Appl., 82(1994), pp. 315-321.

[3] V. LAKSHMikANTham, Further improvement of Generalized quasilinearization, Nonlinear Anal., 27(1996), pp. 223-227.

[4] V. Lakshmikantham and A.S. Vatsala, Generalized Quasilinearization for Nonlinear Problems, Kluwer Academic Publishes, Dordrecht, 1998.

[5] J.J. NiETo, Generalized quasilinearization method for a second order differential equation with Dirichlet boundary conditions, Proc. Amer.Math. Soc., 125(1997), pp. 2599-2604.

[6] A. Cabada, J.J. Nieto and Rafael Pita-Da-veige, A note on rapid convergence of approximate solutions for an ordinary Dirichlet problem, Dynamics of Continuous, Discrete and impulsive systems, 4(1998), pp. 23-30.

[7] A. Cabada AND J.J. Nieto, Quasilinearization and rate of convergence for higher order nonlinear periodic boundary value problems, J. Optim. Theory Appl., 108(2001), pp. 97-107.

[8] Bashir Ahmad, J.J. Nieto and N. Shahzad, The Bellman-Kalaba-Lakshmikantham quasilinearization method for Neumann problems, J. Math. Anal. Appl., 257(2001), pp. 356-363.

[9] Bashir Ahmad, J.J. Nieto and N. Shahzad, Generalized quasilinearization method for mixed boundary value problems, App. Math. Comp., 133(2002), pp. 423-429.

[10] J.O. Adeyeye ANd U.U. Livinus, An extension of the generalized quasilinearization method of Lakshmikantham, Nonlinear Studies 10(2003), pp. 195-199.

[11] W. Coppel, Disconjugacy, Lecture Notes in Mathematics, Vol.220, Springer-Verlag, NewYork/Berlin (1971).

[12] I.T. Kiguradze And A.G. Lomtatidze, On certain boundary value problems for second-order linear ordinary differential equations with singularities, J. Math. Anal. Appl., 101(1984), pp. 325-347.

[13] C.P. Gupta, A second order m-point boundary value problem at resonance, Nonlinear Anal., $24(1995)$, pp. $1483-1489$.

[14] C.P. Gupta And S. Trofimchuck, A priori estimates for the existence of a solution for a multi-point boundary value problem, J. Inequal. Appl., 5(2000), pp. 351-365.

[15] P. Eloe AND Y. GaO, The method of quasilinearization and a three-point boundary value problem, J. Korean Math. Soc., 39(2002), pp. 319-330. 